\title{
Antiprogesterone Therapy
}

National Cancer Institute

\section{Source}

National Cancer Institute. Antiprogesterone Therapy. NCI Thesaurus. Code C15521.

Drug treatment to block the action of the female hormone progesterone. 